\title{
Risk and Protective Factors for Prospective Changes in Adolescent Mental Health during the COVID-19 Pandemic
}

\author{
Natasha R. Magson $\mathbb{1}^{1} \cdot$ Justin Y. A. Freeman ${ }^{1} \cdot$ Ronald M. Rapee ${ }^{1} \cdot$ Cele E. Richardson ${ }^{1,2} \cdot$ Ella L. Oar ${ }^{1}$. \\ Jasmine Fardouly ${ }^{1}$
}

Received: 9 September 2020 / Accepted: 13 October 2020 / Published online: 27 October 2020

(c) Springer Science+Business Media, LLC, part of Springer Nature 2020

\begin{abstract}
The restrictions put in place to contain the COVID-19 virus have led to widespread social isolation, impacting mental health worldwide. These restrictions may be particularly difficult for adolescents, who rely heavily on their peer connections for emotional support. However, there has been no longitudinal research examining the psychological impact of the COVID-19 pandemic among adolescents. This study addresses this gap by investigating the impact of the COVID-19 pandemic on adolescents' mental health, and moderators of change, as well as assessing the factors perceived as causing the most distress. Two hundred and forty eight adolescents $\left(M_{\text {age }}=14.4 ; 51 \%\right.$ girls; $81.8 \%$ Caucasian $)$ were surveyed over two time points; in the 12 months leading up to the COVID-19 outbreak (T1), and again two months following the implementation of government restrictions and online learning (T2). Online surveys assessed depressive symptoms, anxiety, and life satisfaction at T1 and T2, and participants' schooling, peer and family relationships, social connection, media exposure, COVID-19 related stress, and adherence to government stay-at-home directives at T2 only. In line with predictions, adolescents experienced significant increases in depressive symptoms and anxiety, and a significant decrease in life satisfaction from T1 to T2, which was particularly pronounced among girls. Moderation analyses revealed that COVID-19 related worries, online learning difficulties, and increased conflict with parents predicted increases in mental health problems from T1 to T2, whereas adherence to stay-at-home orders and feeling socially connected during the COVID-19 lockdown protected against poor mental health. This study provides initial longitudinal evidence for the decline of adolescent's mental health during the COVID-19 pandemic. The results suggest that adolescents are more concerned about the government restrictions designed to contain the spread of the virus, than the virus itself, and that those concerns are associated with increased anxiety and depressive symptoms, and decreased life satisfaction.
\end{abstract}

Keywords Adolescence $\cdot$ COVID-19 $\cdot$ Depressive symptoms $\cdot$ Anxiety $\cdot$ Life satisfaction $\cdot$ Longitudinal

\section{Introduction}

The COVID-19 pandemic has swept through the globe quickly and indiscriminately. Government restrictions put in place to slow the spread of the virus have led to widespread social isolation, which can have profound consequences for mental health (Brooks et al. 2020). While

Natasha R. Magson

Natasha.Magson@mq.edu.au

1 Centre for Emotional Health, Department of Psychology, Macquarie University, Sydney, New South Wales, Australia

2 School of Psychological Science, University of Western Australia, Perth, Western Australia, Australia these restrictions have been challenging for people of all ages, they may be particularly difficult for adolescents, who at this developmental stage rely heavily on their peer connections for emotional support and social development (Ellis and Zarbatany 2017). As orders to stay at home and socially distance from others impedes most peer interaction, it is important to examine the impact that this is having on adolescents' mental health, especially given the strong associations between interpersonal stress and the onset of emotional difficulties among adolescents (Rapee et al. 2019). However, to date there is no longitudinal research examining the psychological impact of the COVID-19 pandemic among adolescents, and what is known is limited by retrospective reports of perceived mental health changes (e.g., Hawke et al. 2020). The current study addressed this gap by examining changes in adolescent mental health 
within a longitudinal framework that included baseline measures of adolescents' mental health before the COVID19 pandemic, and a second measure two months following the implementation of government restrictions and online learning.

\section{Increased Vulnerability during Adolescence}

To appreciate the importance of examining the effects of the COVID-19 pandemic on adolescent mental health, one must first understand the pertinent developmental changes that occur during adolescence that may make this a particularly distressing time. Adolescence has often been labeled by developmental theorists as a period of storm and stress (Casey et al. 2010). This is due in part, to the physical and chemical changes occurring in the brain from early adolescence, which result in a 'neural mismatch' whereby emotionality is heightened in response to real and/or perceived stressors (Bailen et al. 2019), yet the self-regulatory system required to manage these emotions remains largely underdeveloped until early adulthood (Somerville et al. 2010). Another defining characteristic of adolescence is the marked increase in social sensitivity and the importance of peers (Somerville 2013). As adolescents strive for independence from their parents, the time spent with peers increases dramatically, and for the first time, friends rather than parents become the primary source of interaction and influence (Meuwese et al. 2017). However, the increased social sensitivity that emerges during adolescence also means that peer relationships can be a major source of conflict, rejection, and interpersonal stress (Somerville 2013). During adolescence, acceptance and rejection by peers is used to guide behaviors, shape self-concept and gauge self-worth (Connell and Wellborn 1991). Thus, negative peer interactions during this important developmental window can lead to poor self-concept, a low sense of worth, and subsequent increases in symptoms of anxiety and depression, whereas positive peer relationships can provide social and emotional support, which are known to protect against the risk of both depression and anxiety (La Greca and Harrison 2005).

The increases in interpersonal stress, coupled with heightened emotional reactivity and low emotion-regulation, can place adolescents at a greater risk of developing many common forms of psychopathology including generalized anxiety, eating disorders, depression, and social anxiety (Rapee et al. 2019). In fact, this particular subset of internalizing disorders has previously been referred to as "disorders of adolescence" due to their typical median onset age of 13 to 19 years (Rapee et al. 2019). Collectively, the developmental characteristics of adolescence, the typical age of onset for these social emotional disorders, and the fact that adolescents have been conducting their schooling online, spending most of their time indoors and are physically separated from their peers, means they may be at an increased risk of developing psychological problems during the COVID-19 global pandemic.

\section{Previous COVID-19 Studies}

Whilst it may take years, and many studies, to fully understand the sequelae of the COVID-19 pandemic, prior research shows a consistent link between the pandemic and mental health. Importantly, existing research highlights a number of COVID-19 related factors (e.g., government restrictions, media exposure, etc.) that may influence these associations. As the current study explores these factors in conjunction with prospective changes in mental health, the existing research is reviewed here briefly to demonstrate the association between COVID-19 and mental health, and to highlight any relevant influencing factors.

Previous studies with adults consistently point to the detrimental impact of COVID-19 on the mental health of individuals. For example, research conducted among 1210 respondents during the early stages of the crisis in China found that more than half $(53.8 \%)$ rated the negative impact of the COVID-19 pandemic on their psychological health as moderate to severe (Wang et al. 2020). A second study in China reported COVID-19 related increases in generalized anxiety, which were found to be more pronounced in younger people ( $<35$ years) compared to older age groups (Huang and Zhao 2020). A meta-analysis of 13 studies of mental health among healthcare workers found that almost a quarter exhibited elevated COVID-19 related symptoms of anxiety $(23.2 \%)$ and depression $(22.8 \%)$, and more than a third $(38.9 \%)$ experienced problems with insomnia (Pappa et al. 2020).

To date, few studies have evaluated the impact of the pandemic on mental health in adolescents. One study surveyed 1738 Chinese participants at two time points following the commencement of the pandemic and found no significant changes across time in depression, anxiety and stress over the study period (Wang et al. 2020). However, there was some indication that younger people (aged 12-21 years) perceived the impact of COVID-19 to be greater than older adults (aged 50-59 years). Beliefs about the ease of virus transmission and increased media exposure to health information about COVID-19 were significantly associated with depression and/or anxiety at both time points. In contrast, taking precautionary measures to avoid transmission (e.g., regular handwashing), complying with the stayat-home orders, and low perceived likelihood of contracting and dying from COVID-19 were all found to be protective.

In a study focused on youth, retrospective reports of mental health symptoms three months prior to the pandemic were compared to those three weeks into the pandemic 
among 622 Canadian adolescents and young adults (Hawke et al. 2020). Participants perceived reductions in their mental health across the period, particularly in depression/ low mood and anxiety. Another study also found high levels of depression and anxiety in Chinese adolescents during the peak of the pandemic, although lower levels were reported among males and youth that regularly engaged in exercise (Chen et al. 2020). Finally, a survey of 1054 Canadian adolescents $\left(M_{\mathrm{age}}=16.68\right)$ found that adolescents were most concerned about the impact on their schooling, followed by general concerns about the COVID19 crisis, and not feeling connected to friends (Ellis et al. 2020). Further, COVID-19 related concerns, spending less time on schoolwork and more time with friends was positively associated with depression, whereas spending more time with family was negatively associated. COVID-19 related concerns were also positively associated with loneliness, while spending more time with family and friends, and engaging in more physical activity, was negatively associated. Social media use moderated the association between COVID-19 concerns and depression, with those reporting high levels of social media use also reporting the most depression.

\section{Potential Risk and Protective Moderating Factors}

The cross-sectional research cited above points to a number of factors that may be important for examining the association between adolescent mental health and COVID-19 longitudinally. Namely age, gender, media exposure, family conflict, changes to schooling, adherence to restrictions, and levels of social connection have all been implicated as potential moderators within the existing research. As previous research has demonstrated that adolescents were particularly concerned about feeling socially disconnected from their friends, and that COVID-19 related worries along with less time on schooling were significantly associated with depression cross-sectionally (Ellis et al. 2020), it is possible that social connectedness, COVID-19 related concerns, and disruptions to schooling may predict change from pre-pandemic to intra-pandemic levels of depression, anxiety and life satisfaction. Further, as a prior two-wave study found that increased exposure to media reports about COVID-19 was significantly associated with increases in anxiety and depression, whereas complying with the stay at home rules was protective (Wang et al. 2020), it is important to examine these associations longitudinally. Considering the substantial amount of time adolescents spend on social media, it may also be important to examine the effect of exposure to COVID-19 related media reports via social media, particularly as prior has found that increased social media use during the pandemic was associated with increased adolescent depression and anxiety (Ellis et al. 2020).
Interestingly none of the studies reviewed above investigated whether mental health outcomes during the COVID-19 pandemic differed by sex, which is surprising considering the wellestablished sex differences in the prevalence and severity of depression and anxiety (Rose and Rudolph 2006). Hence, research examining sex differences in adolescents' responses to COVID-19 and its associated restrictions is needed to address this gap. Finally, as depression and anxiety are known to increase, and life satisfaction to decrease, from early adolescence onward (Goldbeck et al. 2007; Rapee et al. 2019), it is important to ensure that any potential prospective changes in mental health symptoms during COVID-19 are not simply due to age effects. However to date, developmental differences in COVID-19 responses have not been examined in adolescent samples.

\section{The Current Study}

Taken together, the above research provides consistent evidence that the COVID-19 crisis may be having a significant impact on the psychological health of adolescents across the globe. However, all of these studies have relied on respondents indicating the perceived impact of COVID19 on their current mental health. Only longitudinal studies that include a baseline measure before COVID-19 can truly detect changes in mental health related to the pandemic, although there has been no such research to date. To address this gap, the primary purpose of the current research was to determine the effect of the pandemic and the governmentimposed restrictions associated with the response to COVID-19 on the emotional health of adolescents. The first aim was to identify which factors were viewed by adolescents as producing the greatest COVID-19 related distress. It was hypothesized that not being able to see and spend time with friends, and concerns about moving to online learning, would cause adolescents the most distress. The second aim was to prospectively investigate the impact of the COVID-19 pandemic on changes in adolescent anxiety, depressive symptoms, and life satisfaction. It was anticipated that from T1 (pre-pandemic) to T2 (during the pandemic) adolescents would report an increase in symptoms of anxiety and depression, and a decrease in overall life satisfaction. The third aim was to ascertain which factors during the pandemic served to decrease or increase the risk of experiencing adjustment difficulties two months after the pandemic began and whether there were any age or sex differences evident. It was predicted that disruptions to schooling, COVID-19 related distress, family conflict, and frequent media exposure during the pandemic, would serve to increase the risk of mental health problems, whereas feeling socially connected and adhering to COVID-19 related restrictions during the lockdown would decrease the risk of mental health problems two months into the 
pandemic. As depression and anxiety are more prevalent in girls than in boys, it was expected that any changes in mental health symptoms from $\mathrm{T} 1$ to $\mathrm{T} 2$ would be stronger in girls compared to boys, and if the predicted increases in mental health problems from $\mathrm{T} 1$ to $\mathrm{T} 2$ are simply due to developmental maturation, it was expected older adolescents would report greater increases in symptoms of depression and anxiety, and greater decreases in life satisfaction, than younger adolescents.

\section{Methods}

\section{Participants}

Participants in the current study were part of the larger longitudinal Risks to Adolescent Wellbeing Project (the RAW Project) who have been completing online questionnaires annually for the past four years. Participants in the study resided in an urban area of New South Wales (NSW) Australia, and the majority of participating families in the RAW Project are Caucasian (81.8\%), speak English as a first language (96.4\%), and have previously reported a middle to high socioeconomic status (79.2\%). Of the 467 adolescents invited to participate in the COVID-19 survey, $248(53 \%)$ returned completed responses and thus comprised the current sample. At the time the COVID-19 survey was completed, the adolescents were aged 13 to 16 years $\left(M_{\text {age }}=14.4, S D=0.5\right)$, with almost equal numbers of boys $(n=122)$ and girls $(n=126)$ participating.

\section{Measures}

\section{Generalized anxiety}

The Generalized Anxiety subscale (e.g., "I worry about things") of the Spence Children's Anxiety Scale (SCAS-C; Spence 1998) was used to measure self-reported symptoms of anxiety. Adolescents were required to indicate how often they experienced each symptom on a 4-point Likert scale ranging from 0 (never) to 3 (always). The total scores for the Generalized Anxiety subscale ranged from 0 to 18 with higher scores indicating greater anxiety. The Cronbach's alphas in the current study were acceptable ( $\alpha=0.86$ at T1 and 0.87 at T2).

\section{Depressive symptoms}

The Short Mood and Feelings Questionnaire-Child Version (SMFQ-C; Angold et al. 1995) is a 13-item self-report measure used to assess symptoms of depression in children and adolescents. Participants were required to indicate how true each statement was for them over the past 2 weeks on a 3-point Likert scale ranging from 0 (not true) to 2 (always true). Total scores ranged from 0 to 26 with higher scores indicating more depressive symptoms. The reliability of the measure was good in the current sample ( $\alpha=0.91$ at T1 and 0.93 at T2).

\section{Life satisfaction}

The Student's Life Satisfaction Scale (SLSS; Huebner 1994) is a 9-item self-report measure of global life satisfaction for young people. Adolescent participants were required to indicate their level of agreement with a statement (e.g., "My life is going well") on a 7-point Likert scale ranging from 1 (strongly disagree) to 7 (strongly agree). After reverse coding the two negatively worded items, item scores were averaged to provide a mean score ranging from 1 to 7 , with higher scores indicating greater life satisfaction. The Cronbach's alphas in the current study were high $(\alpha=0.91$ at $\mathrm{T} 1$ and 0.92 at $\mathrm{T} 2$ ).

\section{COVID-19 related distress}

This 18-item measure assessing COVID-19 related distress was specifically developed for the current research (items listed in Fig. 1). Adolescents were required to indicate how distressed they were about each item listed on a 10-point scale ranging from 1 (not at all distressed) to 10 (extremely distressed). Item scores were averaged to provide a mean score ranging from 1 to 10 with higher scores indicating greater COVID-19 related distress. The Cronbach's alpha for the new scale was 0.91 .

\section{Disruption to schooling}

To understand the impact of COVID-19 on schooling four items were developed to assess: format of school attendance, difficulties during online learning, motivation to complete school work, and impact on education. Specifically, adolescents were asked if they were (a) attending school in-person; (b) learning online only; or (c) participating in a mix of in-person and online schooling. For those learning online, an additional yes or no question was asked: "Have you had any difficulties switching to online learning?", and if yes, an open-ended question followed asking for details of their difficulties. The self-perceived impact of online learning and COVID-19 on education was measured using two questions, "Do you think your education is suffering due to online schooling?" and "Do you think your education is suffering due to the disruptions from COVID19?", rated on a 4-point scale from 1 (not at all) to 4 (a great deal). The two questions were highly correlated $(r=0.758$, $p<0.001$ ) so a mean of the two scores was calculated with higher scores indicating a larger impact on schooling. A single-item question was used to measure the change in students' motivation to complete school work (i.e., 
COVID-19 Related Distress

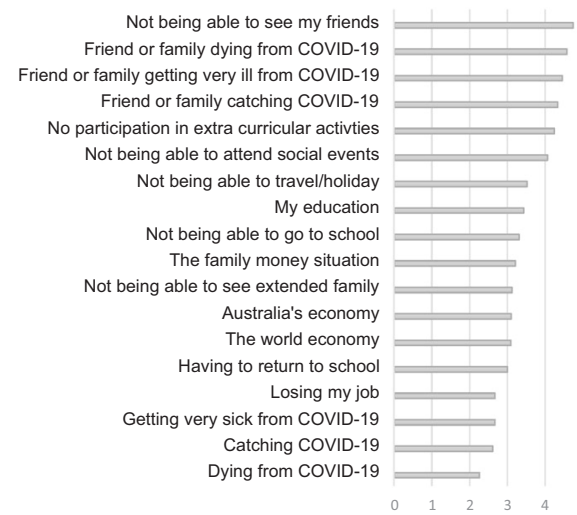

Fig. 1 Factors contributing to COVID-19 related distress among adolescents. Rated on a scale of 0 (not at all distressed) to 10 (extremely distressed)

"Compared to how motivated you usually are to do school work, how motivated do you feel now to do your school work?") rated on a 3 -point scale $(1=$ much less motivated, $2=$ about the same, $3=$ much more motivated).

\section{Media exposure}

One question assessed their exposure to traditional news media (i.e., "On average, how often to you read/watch media reports about COVID-19 each day?") and a second question assessed social media related exposure (i.e., "On average, how often do you read posts about COVID-19 on social media each day?"). Both items were rated on a 6point scale from 1 (not at all) to 6 (five times or more).

\section{Interpersonal conflict}

The current study developed four self-report item to assess change in interpersonal conflict between adolescents and their mothers, fathers, siblings, and friends due to the COVID-19 social distancing rules and stay at home restrictions (e.g., "Do you feel you are having more or fewer disagreements/arguments with your father?"). Adolescents responded on a 5-point Likert scale ranging from 1 (we have argued much less) to 5 (we have argued much more), thus higher scores indicated greater interpersonal conflict.

\section{Social connectedness}

The Social Connectedness Scale (SCS; Lee and Robbins 1995) is a measure of how emotionally distant or connected a respondent feels with those close to them, and society more broadly. Adolescents were required to indicate their level of agreement with eight statements (e.g., "I feel distant from people") on a 6-point scale, ranging from 1 (strongly disagree) to 6 (strongly agree). Item scores were averaged to provide a mean score ranging from 1 to 6 , with higher scores indicating greater social connectedness. The Cronbach's alpha value in the current study was high $(\alpha=0.91)$.

\section{Adherence to COVID-19 Australian government stay-at- home directive}

At the time T2 data was collected, laws in Australia were in place to help minimize the spread of COVID-19. Residents of the state New South Wales were asked to stay at home unless they were going to work or school, obtaining food or other essential goods and services, exercising, or seeking medical care. To assess adherence to this stay-at-home directive, adolescents were asked how often they had left their home for reasons other than those listed above, responding on a 5-point scale from 0 (I have only left my home for the reasons listed above) to 4 (more than 10 times).

\section{Procedure}

The parents of all adolescents currently enrolled in the RAW Project were sent information about the COVID-19 study and a link to the T2 online survey. Parents who consented to their child participating, then shared the link with their child who assented online prior to beginning the survey. Participants were asked to complete the survey within two weeks of receiving the initial email, after which the survey was closed. The World Health Organization declared COVID-19 a pandemic on March 11, 2020. The T1 data used for the current study was collected online throughout 2019 as part of the larger RAW Project (thus prior to the COVID-19 pandemic), and the T2 data was collected between May 5th and May 14th 2020, approximately two months after the Australian government had imposed the stay-at-home orders and schools had moved to online learning.

\section{Data Analysis}

Distribution and descriptive statistics were obtained through SPSS (v26; IBM Corp. 2019). To determine whether levels of depressive symptoms, anxiety, and life satisfaction significantly increased or decreased in the period before the pandemic began and two months into the pandemic, a paired samples $t$-test in SPSS was conducted. Potential moderators of the change in mental health scores before and during COVID-19 were examined in SPSS using Model 2 in the MEMORE macro (Montoya and Hayes 2017), which is specifically designed to assess moderation in two instance repeated measures research designs (Montoya 2019). This procedure computes a pre-post difference score and then determines whether the moderator of interest predicts that difference (Judd et al. 2001; Kenny 2018). For all non-dichotomous moderators, significant interactions 
Table 1 Means and standard deviations for key study variables for male, female, and total sample

\begin{tabular}{|c|c|c|c|c|c|c|c|c|}
\hline & \multicolumn{2}{|l|}{ Males } & \multicolumn{2}{|c|}{ Females } & \multicolumn{4}{|c|}{ Total sample } \\
\hline & Mean & SD & Mean & SD & Mean & SD & Skew & Kurtosis \\
\hline Generalized anxiety $\mathrm{T} 1$ & 3.63 & 3.13 & 5.55 & 4.05 & 4.60 & 3.74 & 1.13 & 1.12 \\
\hline Generalized anxiety $\mathrm{T} 2$ & 3.64 & 3.16 & 6.52 & 4.31 & 5.10 & 4.05 & 0.72 & -0.01 \\
\hline Depressive symptoms T1 & 2.81 & 3.18 & 4.77 & 5.00 & 3.81 & 4.31 & 1.57 & 2.21 \\
\hline Depressive symptoms T2 & 4.02 & 4.76 & 8.16 & 6.46 & 6.12 & 6.04 & 1.09 & 0.41 \\
\hline Life satisfaction $\mathrm{T} 1$ & 5.65 & 0.87 & 5.50 & 1.09 & 5.57 & 0.99 & -0.81 & 0.29 \\
\hline Life satisfaction $\mathrm{T} 2$ & 5.23 & 1.10 & 4.81 & 1.14 & 5.01 & 1.14 & -0.52 & -0.25 \\
\hline COVID-19 distress T2 & 3.22 & 1.48 & 3.70 & 1.47 & 3.46 & 1.49 & 0.50 & -0.27 \\
\hline Impact on education $\mathrm{T} 2$ & 2.10 & 0.79 & 2.10 & 0.74 & 2.10 & 0.76 & 0.90 & 0.72 \\
\hline Motivation to study $\mathrm{T} 2$ & 1.66 & 0.68 & 1.57 & 0.67 & 1.62 & 0.68 & 0.64 & -0.67 \\
\hline Traditional media exposure $\mathrm{T} 2$ & 2.10 & 1.16 & 1.85 & 0.90 & 1.97 & 1.04 & 1.72 & 3.76 \\
\hline Social media exposure $\mathrm{T} 2$ & 2.26 & 1.48 & 2.25 & 1.38 & 2.26 & 1.42 & 1.32 & 1.10 \\
\hline Conflict-mother $\mathrm{T} 2$ & 3.05 & 0.97 & 2.86 & 1.08 & 2.95 & 1.03 & -0.14 & -0.07 \\
\hline Conflict-father T2 & 3.13 & 0.95 & 2.95 & 1.09 & 3.04 & 1.03 & -0.28 & -0.03 \\
\hline Conflict—siblings T2 & 3.43 & 0.99 & 3.40 & 1.11 & 3.41 & 1.05 & -0.48 & -0.13 \\
\hline Conflict—friends $\mathrm{T} 2$ & 2.31 & 0.94 & 2.30 & 1.07 & 2.30 & 1.00 & -0.01 & -1.10 \\
\hline Social disconnection $\mathrm{T} 2$ & 2.56 & 1.08 & 3.26 & 1.38 & 2.92 & 1.29 & 0.57 & -0.18 \\
\hline Stay at home adherence $\mathrm{T} 2$ & 1.52 & 0.76 & 1.44 & 0.64 & 1.48 & 0.70 & 1.40 & 1.54 \\
\hline
\end{tabular}

were probed at $1 \mathrm{SD}$ above and below the mean. Data coverage for T1 and T2 mental health variables was $99.6 \%$ to $100 \%$, respectively. All other variables contained less than $1 \%$ missing data with the exception of the family conflict variables which had a "not applicable option" for family members not living within the same household. This resulted in non-responses of $3.2 \%$ for mother, $9.7 \%$ for father, and $7.7 \%$ for siblings. Due to the small percentage of missing data, all analyses were conducted using pairwise deletion.

\section{Results}

\section{Preliminary Results}

Descriptive statistics (for total sample, and boys and girls separately) and correlations between key variables are displayed in Tables 1 and 2. As shown in Table 1, most variables were normally distributed with no obvious skewedness ( -0.81 to 1.72 ), although the kurtosis of values of depressive symptoms at T1 (2.21) and exposure to traditional media at T2 (3.76) fell slightly above the recommended values of plus or minus two (Gravetter et al. 2020). As expected, on average girls reported more symptoms of depression and anxiety at both time points, whereas boys reported more familial conflict during the lockdown period. Boys and girls were more similar on average levels of life satisfaction, exposure to COVID-19 related media, and attitudes toward the impact of COVID-19 on their schooling. The correlations in Table 2 show that all mental health variables were significantly correlated within and between time points in the predicted direction. COVID-19 related distress and feeling socially disconnected from others were most strongly related to all mental health symptoms, as well as being significantly associated with viewing posts about COVID-19 on social media, less motivation to study, a greater perceived negative impact on schooling, and greater conflict with siblings.

\section{Adolescents Distress during COVID-19}

Addressing the first aim concerning what is causing adolescents the most distress, Fig. 1 shows that adolescents reported low to moderate levels of COVID-19 related stress across the 18 items assessed. As predicted, the most distressing issue for adolescents during this time was not being able to see their friends, closely followed by a friend or family member contracting and getting very sick and/or dying from COVID-19. Also high on adolescents' lists of concerns was the inability to participate in their normal extra-curricular activities (e.g., sports, dance, music lessons etc.) or attend social events. In contrast, they reported very little concern about contracting, getting ill or dying from COVID-19 themselves.

\section{Overall Changes in Mental Health}

To address the second aim relating to changes in mental health prior to and during the pandemic, the results of the paired samples $t$-tests showed significant increases in 
50

Journal of Youth and Adolescence (2021) 50:44-57

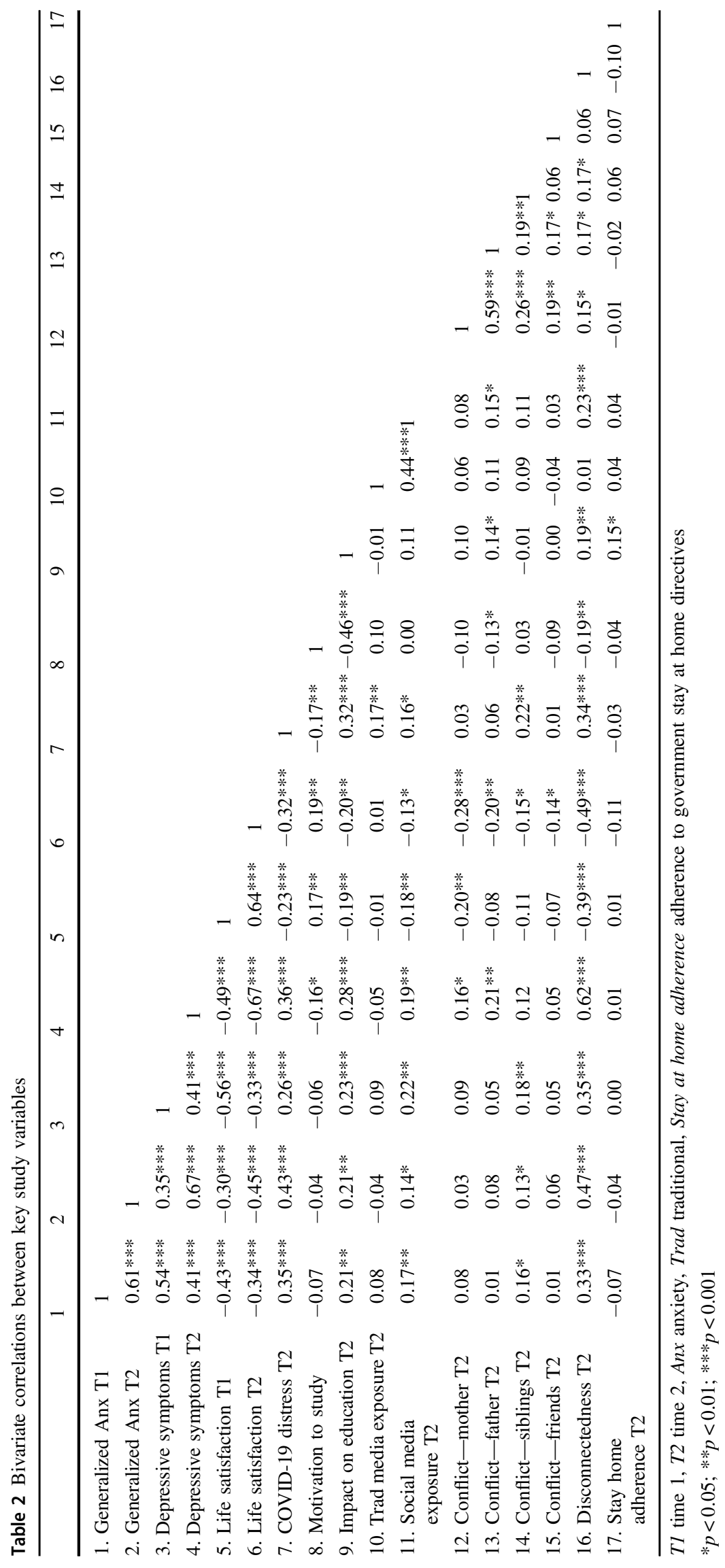

Springer 


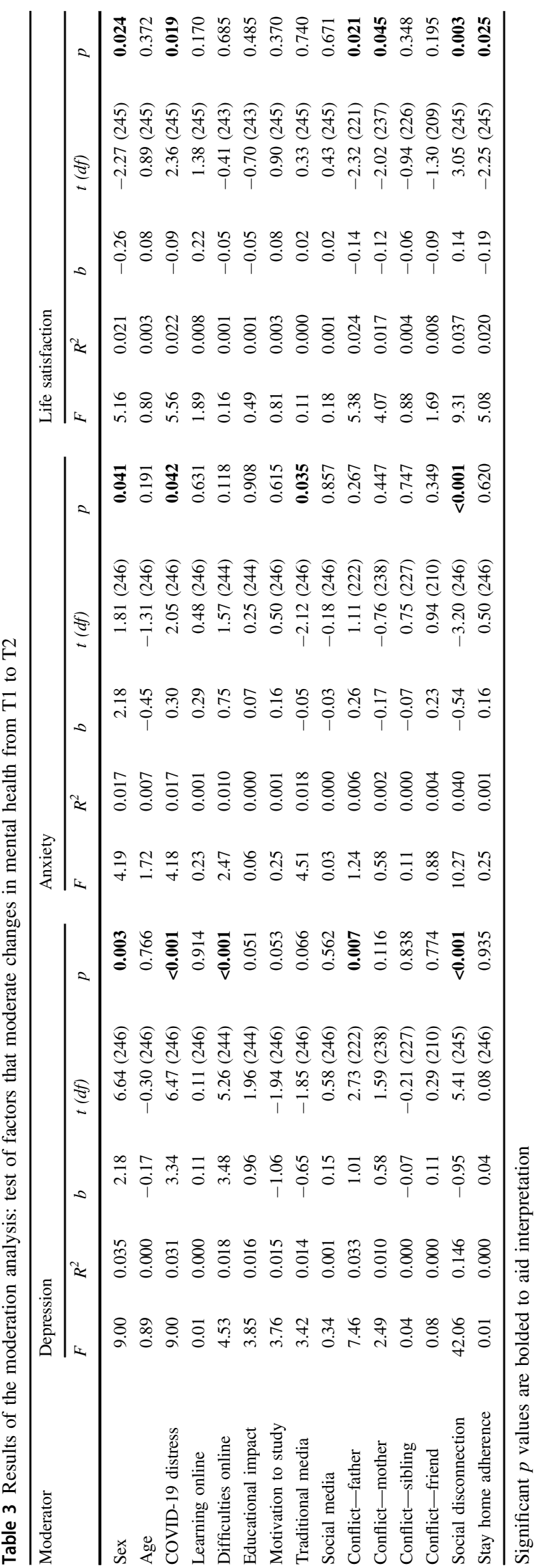

adolescents' symptoms of depression, $(t(1,247)=6.26, p<$ $0.001, d=0.15)$, and anxiety, $(t(1,244)=5.26, p<0.001$, $d=0.40)$, and a significant decrease in life satisfaction, $(t(1$, $244)=-5.26, p<0.001, d=0.61$ ) from $\mathrm{T} 1$ (before the pandemic) to T2 (2 months into the pandemic).

\section{Potential Moderating Factors}

To address the third aim of the research, the results for each of the potential moderating factors examined are presented below. To aid interpretation, the statistical results of all moderating analyses are summarized in Table 3 and described in text below.

\section{Sex}

Sex of the youth significantly moderated the change scores in depressive symptoms, with results indicating that although there were significant increases in depressive symptoms from $\mathrm{T} 1$ to $\mathrm{T} 2$ for both boys and girls, this effect was more pronounced in girls. This result was replicated for changes in anxiety, with girls again showing the largest increases in symptoms from T1 to T2. Finally, sex significantly moderated the change in life satisfaction from $\mathrm{T} 1$ to $\mathrm{T} 2$ with girls showing the greatest decrease.

\section{Age}

Age did not moderate change in depressive symptoms, anxiety, or life satisfaction from $\mathrm{T} 1$ to $\mathrm{T} 2$.

\section{COVID-19 related distress}

Levels of distress associated with the COVID-19 pandemic significantly moderated change scores in depressive symptoms, anxiety, and life satisfaction from T1 to T2. Specifically, adolescents with high and moderate levels of COVID19 related distress experienced significantly greater increases in anxiety and depressive symptoms, and significantly greater decreases in life satisfaction, over time than those with low levels of COVID-19 related distress.

\section{Disruption to schooling}

The most commonly reported problems when learning online were related to technology problems, not understanding the learning materials, being unable to ask the teacher to explain the work, and problems with motivation. Results showed that switching to learning exclusively online had no significant effect on levels of depressive symptoms, anxiety, or life satisfaction. However, experiencing the abovementioned difficulties with online learning did significantly moderate change in depressive symptoms, 
with those who experienced problems reporting significantly more depressive symptoms during COVID-19, compared to those who experienced no problems with online learning. Online learning difficulties did not moderate changes in anxiety or life satisfaction from $\mathrm{T} 1$ to $\mathrm{T} 2$. Participants' perceptions that their education was suffering during the COVID-19 lockdown had no significant effect on change scores in depressive symptoms, anxiety or life satisfaction. Finally, differences in motivation to engage in school work before and during the COVID-19 pandemic had no significant effect on changes in depressive symptoms, anxiety or life satisfaction.

\section{Media exposure}

Frequency of exposure to traditional media reports (i.e., television, newspaper) about COVID-19 had no significant effect on change in depressive symptoms, but there was a significant moderating effect on changes in anxiety. Results showed those reporting high levels of traditional media exposure had significantly greater decreases in anxiety from T1 to T2 than those reporting low exposure. Frequency of exposure to COVID-19 traditional media reports had no significant effect on changes in life satisfaction. The frequency of reading posts about COVID-19 on social media had no significant effect on changes in depressive symptoms, anxiety or life satisfaction.

\section{Interpersonal conflict}

Increased conflict with siblings, friends and mothers had no significant interaction effect on depressive symptoms, however, increased conflict with fathers during COVID-19 significantly moderated change in depressive symptoms from $\mathrm{T} 1$ to $\mathrm{T} 2$. Those experiencing an increase in conflict with their fathers reported more depressive symptoms at $\mathrm{T} 2$ than those that did not. Levels of interpersonal conflict with siblings, friends, mothers or fathers did not moderate change scores in anxiety. Interpersonal conflict with both mothers and fathers significantly moderated changes in life satisfaction with those reporting greater conflict with their mother and father also reporting larger declines in life satisfaction than those with low parental conflict. Interpersonal conflict with siblings and friends had no effect on changes in life satisfaction.

\section{Social connectedness}

Feeling socially connected during the COVID-19 lockdown period significantly moderated changes in depressive symptoms, anxiety and life satisfaction. Those perceiving high levels of social connection during COVID-19 reported significantly fewer depressive symptoms and anxiety, and significantly more life satisfaction from $\mathrm{T} 1$ to $\mathrm{T} 2$ than those feeling socially disconnected during the lockdown period.

\section{Adherence to COVID-19 government restrictions}

Adherence to the government's stay at home directive had no significant effect on changes in depressive symptoms or anxiety, however, those reporting greater adherence to the stay at home rules reported lower declines in life satisfaction from $\mathrm{T} 1$ to $\mathrm{T} 2$ compared to those who continued to leave their homes more frequently.

\section{Discussion}

The COVID-19 pandemic has had a significant impact on the lives of millions around the world. Government measures put in place to contain the virus have physically and socially isolated adolescents from their friends, extended family, and other social support networks, leaving many feeling socially isolated, and consequently, at an increased risk of psychological disorder. However, to date, there has been no longitudinal research examining how the pandemic has impacted adolescents' mental health despite decades of research demonstrating the potential lifelong risks associated with prolonged interpersonal stress and social isolation during the adolescent years (e.g., Loades et al. 2020). Further, research has not yet examined which demographic and COVID-19 related factors serve to increase or decrease adolescent mental health symptoms over time. The current study addressed these research gaps by conducting a longitudinal investigation into the effect the COVID-19 virus and its associated restrictions are having on adolescents' mental health, as well as identifying some of the critical impacts and life changes associated with the pandemic. Potential moderators of change in mental health symptoms were also investigated including age, sex, disruptions to schooling, COVID-19 related distress, family conflict, media exposure, social connection, and adherence to COVID-19 related restrictions.

\section{Negative Emotionality}

As predicted, the results showed that adolescents' mental health had slightly deteriorated compared to pre-pandemic levels and a number of pandemic-related factors were related to those changes. When interpreting the results, it must be kept in mind that, although mental health measures were taken on two occasions, potential moderators were only measured once and therefore, the results are unable to indicate the direction of relationships.

The pre-pandemic to intra-pandemic increase in depressive symptoms and anxiety, and decrease in life satisfaction 
found in the current study is generally consistent with previous retrospective and cross-sectional studies in both adult and adolescent samples, which have reported perceived increases in depression, anxiety, and loneliness due to the effects of COVID-19 (Chen et al. 2020; Wang et al. 2020; Ellis et al. 2020). The current study strengthens and builds upon this literature by demonstrating that living with the restrictions and concerns surrounding COVID-19 are related to not only increases in emotional distress but also with decreases in life satisfaction. More importantly, the prospective collection of these data allows stronger conclusions to be drawn, that go beyond the perceived attributions of participants. Interestingly, while significant decrements in adolescent mental health were demonstrated prospectively, the size of these effects were quite modest, with changes ranging from 0.2 to 0.6 standard deviations. Clearly a large proportion of adolescents are coping well with the impact of the pandemic, at least in the early stages, and most are showing minimal negative impact.

\section{Gender and Age Differences}

The finding that girls are experiencing greater declines in mental health than boys during the COVID-19 crisis may not only reflect the well-established literature highlighting sex differences in internalizing problems (e.g., Rose and Rudolph 2006), but also that girls are more likely than boys to rely on their social networks for support when dealing with significant life stressors (Tamres et al. 2002). Thus, the move to online schooling and the restrictions placed on social interaction may effectively impede adolescent girls from employing their most commonly used coping strategy. Although this suggestion would need to be formally tested, the current findings suggest that it may be particularly important to encourage adolescent girls to connect with their friendship groups via safe means, so they can continue to access their much-needed social support systems during the COVID-19 crisis. Previous research has also shown that the prevalence of internalizing disorders increases from early to mid-adolescence (Goldbeck et al. 2007). However, the current study did not find any moderating effect of age, suggesting that the observed increase in mental health symptoms from $\mathrm{T} 1$ to $\mathrm{T} 2$ cannot be simply explained by these developmental differences, and may instead be due to the changing social, educational and economic climate as a result of COVID-19.

\section{Social Connection and Familial Conflict}

Consistent with developmental theory stressing the importance of peer relationships and social interaction during adolescence (Meuwese et al. 2017), and previous findings (Ellis et al. 2020), the current results revealed that adolescents' greatest concern during the COVID-19 lockdown was not being able to see their friends. In fact, all items mentioning friends or social connection were rated higher than any of the other COVID-19 related worries. Signifying the possibility of developmental differences, these findings contrast with research in adults, as adults expressed the greatest concern over family members contracting COVID-19 (Wang et al. 2020). The present results also showed that feeling socially disconnected during the pandemic was associated with higher levels of anxiety and depressive symptoms and lower levels of life satisfaction. The importance of feeling socially connected during the pandemic has been demonstrated previously when it was found that adolescents left alone at home all day reported significantly higher levels of depression and anxiety than those who were accompanied by a parent or relative (Chen et al. 2020). As the current results cannot indicate the causal direction, it is equally plausible that emotional distress leads to social disconnection or that social disconnection leads to greater emotional distress. If the latter direction is demonstrated in future research, it might suggest that organizing to have a parent or relative work from home during such stressful times, may help in mitigating the risks for anxiety and depression, as long as it does not result in greater familial conflict.

About a quarter of the adolescents surveyed reported that conflict with their parents had increased during the lockdown period, which was associated with decreases in life satisfaction; and increased conflict with fathers was associated with more depressive symptoms. This increase in parental conflict and decrease in mental health may reflect a developmental discrepancy between adolescents' inherent drive to connect with peers and seek greater autonomy from their parents (Somerville 2013), while at the same time being ordered to stay at home and physically distance from their friends. This possibility is further supported by the finding that although half of the current sample reported an increase in conflict with their siblings, this was not associated with changes to their mental health during the lockdown period.

\section{Educational Concerns}

The current results also indicated that adolescents were not overly concerned with the impact that COVID-19 was having on their education, nor was this related to any of the mental health outcomes examined. These findings are inconsistent with previous work which found that one of adolescents' most pressing COVID-19 related worries was the detriment to their education (Ellis et al. 2020). This may be because the earlier research was carried out immediately following the switch to online learning whereas the current study assessed concerns two months after the initial change, 
providing students with the opportunity to adapt and settle into their new learning environment. It may also be due to the younger age of the current cohort relative to those in the earlier study, most of whom were in senior school-a time when grades are important in determining acceptance into university and future occupational opportunities. Although, there was no association between mental health problems and moving to online learning, the current study did find that experiencing difficulties during online learning was associated with an increase in depressive symptoms from $\mathrm{T} 1$ to T2. The most commonly reported difficulties were related to technology problems, not understanding the learning materials, being unable to ask the teacher questions, and problems with motivation. All of these difficulties could be relatively easily addressed should online learning be continued long-term (e.g., providing a platform to ask teachers questions during the lesson rather than having to wait for an email response), and implementing these changes may mitigate the increased risk for depressive symptoms if the causal direction of this association does go from learning difficulties to depression.

\section{Media Exposure}

Contrary to expectations, high exposure to traditional media reports about COVID-19 had a negative relationship with changes in generalized anxiety levels, and no significant effect on changes in life satisfaction or depressive symptoms. Although there is no clear explanation for the contradictory findings between the current and previous studies, some research suggests that associations between media exposure and internalizing distress are nuanced (Wang et al. 2020). A recent study in China found that although greater depression and anxiety were associated with exposure to radio reports around COVID-19, the same effect was not found in relation to television and internet reports (Wang et al. 2020). These findings are broadly consistent with those found in the current study in that social media posts about COVID-19 were not associated with changes in mental health, whereas reading and watching media reports were positively related to good mental health.

While the direction of these associations cannot be determined from the current results, they are consistent with a suggestion that changes in anxiety led to differences in media exposure. Specifically, due to the established links between avoidant coping and anxiety (Richardson et al. in press), it might be logical to interpret that young people who became more anxious during the COVID-19 period avoided exposure to mainstream media reports. On the other hand, it is possible that watching and reading news reports about the virus plays an important role in building young people's confidence in the public health measures implemented to contain the spread of infection, effectively alleviating uncertainty and concern about its impact. As the rate of transmission and positive cases were fairly low in Australia at the time of the survey, it would be interesting to investigate the role of media exposure further by looking at relationships with adolescent mental health in countries experiencing high rates of transmission and infection, and comparing the findings to those in the current study.

The media also communicates important health information, which may serve to give adolescents a sense of control over what they need to do to avoid contracting the virus, which may also reduce anxiety. Indeed, the current study found those who adhered more closely to government stay-at-home orders reported fewer mental health problems than those who frequently defied them. This is consistent with previous research which also found an association between that taking precautionary measures (e.g., staying at home, wearing a mask) to avoid contracting the virus and lower levels of depression and anxiety (Wang et al. 2020). As with all of the current results, the casual relationship may flow in either direction. On the one hand, these results may suggest that disseminating health information and encouraging adherence to government advice through the media may be an effective way of not only reducing the spread of COVID-19, but also in stabilizing levels of generalized anxiety. On the other hand, the results may suggest that young people who become more distressed by the effects of COVID-19, may be at greater risk to break socially-described precautions, and in turn, may jeopardize their and others' health.

\section{Limitations and Future Directions}

The current study has a number of strengths and limitations. First, this study overcomes many of the limitations of previous research by employing a longitudinal design to allow determination of change in mental health symptoms before and during the COVID-19 pandemic. However, despite the longitudinal data collection on mental health, it is not possible to attribute any changes specifically to the effects of COVID-19 as there was no comparison condition. Similarly, the time two mental health measures and COVID-19 related factors were assessed concurrently, so while it was possible to ascertain that these factors were associated with increases or decreases in mental health, the direction of those associations cannot be determined. Employing alternative methods such as ecological momentary assessment or daily diaries may help to clarify the temporal ordering between social, educational, and emotional experiences during the pandemic and adolescents' mental health. Further, the data were all self-report and thus are subject to bias that may not align with more objective assessments of mental health and behaviors. To address this limitation, future research could incorporate 
parent and teacher reports of adolescents' adjustment during the COVID-19 pandemic. Finally, the sample used in the current study was relatively small and demographically restricted. The sample was predominately of Caucasian ethnicity and included participants within a restricted age range, and perhaps most importantly for a study of the impacts of COVID-19, data were collected within a single country (Australia) and it cannot be ensured that the results are generalizable to adolescents from other countries that differed widely in rates of infection and severity of government-imposed restrictions.

\section{Implications}

While keeping these limitations in mind, several speculative implications can be suggested. First, although it is difficult to disentangle whether the changes in mental health found in the current study are the direct result of anxieties around contracting the virus, or the restrictions put in place to contain it, the current results suggest the latter. Adolescents' concerns around catching and becoming ill from the virus were relatively low whereas difficulties adjusting to the new restrictions placed on social activities and schooling as a result of COVID-19 moderated changes in mental health. Therefore, finding new ways to help adolescents adapt and cope with the changes to their social and physical environment is essential. Ensuring that adolescents, particularly girls, maintain their peer relationships seems especially important, and both parents and teachers should encourage social engagement whenever and however possible, within the confines of government and health guidelines.

In addition, since early changes in symptoms of psychopathology may serve as an antecedent for the onset of chronic mental illness, it is important for parents and school staff to monitor young people for elevated emotional distress and to guide them into early intervention and treatment programs where necessary. It is also recommended that parents and teachers create positive and supportive home and learning environments, both of which have previously been shown to lower levels of internalizing distress in adolescents even when isolated physically from peers (Ellis et al. 2020; Manczak et al. 2019).

Finally, and encouragingly, learning exclusively online did not appear to impact adolescents' mental health, whereas difficulties encountered during online learning did. This demonstrates that adolescents can be resilient and adaptive when needed, as long as they are equipped with the necessary tools and support they require. Most of the online learning difficulties reported were modifiable and related to problems concerning technology, the understanding of learning materials, and being unable to ask the teacher for clarification. Implementing regular question and answer sessions throughout each lesson, finding creative ways to engage students during their online classes, and ensuring students have access to adequate technology may help overcome some of these most common problems, and as a consequence, reduce depressive symptoms associated with online learning difficulties.

\section{Conclusion}

Due to the recency of the COVID-19 pandemic, there is very limited research on its psychological consequences, and no research examining the prospective impact of the virus, and its related restrictions, on the emotional health of adolescents. Thus, this research contributes to the existing literature by demonstrating longitudinal declines in the mental health of adolescents, especially among girls, when comparing pre-pandemic to intra-pandemic levels. The current study contributes further by identifying which COVID-19 related factors served to increase or decrease the risk of adolescents developing mental health problems during the pandemic. Specifically, COVID-19 related worries, difficulties with online learning, and increased family conflict were associated with greater psychological maladjustment. In contrast, greater exposure to traditional media, adherence to government restrictions, and feeling socially connected with others was associated with less distress. Consistent with theory highlighting the importance of peers during the adolescent period, the results showed that adolescents' greatest concerns during the COVID-19 crisis were around the disruption to their social interactions and activities, whereas concerns around contracting or getting ill from the virus were very low. This suggests that it is the restrictions put in place to reduce the spread of the virus, rather than the virus itself, that is causing adolescents the most distress. As social isolation, interpersonal stress, and mental health problems during adolescence can be a precursor for mental health problems across the lifespan, parents and teachers are encouraged to assist adolescents in finding ways to maintain their social networks, monitor young people for signs of emotional distress, provide positive and supportive home and learning environments, and engage with mental health professionals early. Finally, as research on the mental health impact of COVID-19 is still in its infancy, more longitudinal research is needed to gain a greater understanding of the long term implications of this pandemic on the emotional wellbeing of young people.

Acknowledgements The authors extend their gratitude to the many research assistants on the RAW project for carrying out the extensive data collection required for this project and the interns who assisted in the coding of the data sets.

Author's Contributions N.R.M. conceived of the study, prepared the study materials, analyzed the results, and drafted the manuscript; 
J.Y.A.F. helped with the development of the study materials, the coordination of the data collection, and assisted with the data analysis; R.M.R. participated in the study design and helped draft the manuscript; C.E.R. helped design the study and draft the manuscript; E.L.O. helped design the study materials, prepare the data, and draft the manuscript; and J.F. helped design the study materials and draft the manuscript. All authors have read and approved the submitted manuscript.

Funding The Australian Research Council funded this research (grant number FL150100096).

Data Sharing and Declaration The datasets generated and/or analyzed during the current study are not publicly available but are available from the corresponding author upon reasonable request.

\section{Compliance with Ethical Standards}

Conflict of Interest The authors declare that they have no conflict of interest.

Ethical Approval All procedures performed in studies involving human participants were in accordance with the ethical standards of the institutional and/or national research committee and with the 1964 Helsinki declaration and its later amendments or comparable ethical standards.

Informed Consent Informed consent was obtained from all parents of youth participating in the study, and informed assent was obtained from all adolescents.

Publisher's note Springer Nature remains neutral with regard to jurisdictional claims in published maps and institutional affiliations.

\section{References}

Angold, A., Costello, E. J., \& Messer, S. C. (1995). Development of a short questionnaire for use in epidemiological studies of depression in children and adolescents. International Journal of Methods in Psychiatric Research, 5, 237-249.

Bailen, N. H., Green, L. M., \& Thompson, R. J. (2019). Understanding emotion in adolescents: a review of emotional frequency, intensity, instability, and clarity. Emotion Review, 11(1), 63-73.

Brooks, S. K., Webster, R. K., Smith, L. E., Woodland, L., Wessely, S., Greenberg, N., \& Rubin, G. J. (2020). The psychological impact of quarantine and how to reduce it: rapid review of the evidence. The Lancet, 395(10227), 912-920. https://doi.org/10. 1016/S0140-6736(20)30460-8.

Casey, B. J., Jones, R. M., Levita, L., Libby, V., Pattwell, S. S., Ruberry, E. J., \& Somerville, L. H. (2010). The storm and stress of adolescence: insights from human imaging and mouse genetics. Developmental psychobiology, 52(3), 225-235. https://doi. org/10.1002/dev.20447.

Chen, F., Zheng, D., Liu, J., Gong, Y., Guan, Z., \& Lou, D. (2020). Depression and anxiety among adolescents during COVID-19: a cross-sectional study. Brain, Behavior, and Immunity, 88, 36-38. https://doi.org/10.1016/j.bbi.2020.05.061.

Connell, J. P., \& Wellborn, J. G. (1991). Competence, autonomy, and relatedness: a motivational analysis of self-system processes. In Self processes and development. (pp. 43-77). Hillsdale, NJ, US: Lawrence Erlbaum Associates, Inc.
Ellis, W. E., Dumas, T. M., \& Forbes, L. M. (2020). Physically isolated but socially connected: psychological adjustment and stress among adolescents during the initial COVID-19 crisis. Canadian Journal of Behavioural Science/Revue Canadienne des Sciences du Comportement, 52(3), 177.

Ellis, W. E., \& Zarbatany, L. (2017). Understanding processes of peer clique influence in late childhood and early adolescence. Child Development Perspectives, 11(4), 227-232.

Goldbeck, L., Schmitz, T. G., Besier, T., Herschbach, P., \& Henrich, G. (2007). Life satisfaction decreases during adolescence. Quality of Life Research, 16(6), 969-979.

Gravetter, F., Wallnau, L., Forzano, L., \& Witnauer, J. (2020). Essentials of statistics for the behavioral sciences (10 ed.). New York: Cengage Learning.

Hawke, L. D., Barbic, S. P., Voineskos, A., Szatmari, P., Cleverley, K., Hayes, E., ... Cheung, A. (2020). Impacts of COVID-19 on Youth Mental Health, Substance Use, and Well-being: A Rapid Survey of Clinical and Community Samples. The Canadian Journal of Psychiatry, 65(10), 701-709. https://doi.org/10.1177/ 0706743720940562.

Huang, Y., \& Zhao, N. (2020). Generalized anxiety disorder, depressive symptoms and sleep quality during COVID-19 outbreak in China: a web-based cross-sectional survey. Psychiatry Research, 288 (112954). https://doi.org/10.1016/j.psychres.2020.112954.

Huebner, E. S. (1994). Preliminary development and validation of a multidimensional life satisfaction scale for children. Psychological Assessment, 6(2), 149.

IBM Corp. (2019). IBM SPSS statistics software: SPSS statistics V26. Armonk, NY: IBM Corp.

Judd, C. M., Kenny, D. A., \& McClelland, G. H. (2001). Estimating and testing mediation and moderation in within-subject designs. Psychological Methods, 6(2), 115.

Kenny, D. A. (2018). Moderator variables: introduction. http://davida kenny.net/cm/moderation.htm.

La Greca, A. M., \& Harrison, H. M. (2005). Adolescent peer relations, friendships, and romantic relationships: do they predict social anxiety and depression? Journal of Clinical Child \& Adolescent Psychology, 34(1), 49-61. https://doi.org/10.1207/s15374424jccp3401_5.

Lee, R. M., \& Robbins, S. B. (1995). Measuring belongingness: the social connectedness and the social assurance scales. Journal of Counseling Psychology, 42(2), 232.

Loades, M. E., Chatburn, E., Higson-Sweeney, N., Reynolds, S., Shafran, R., Brigden, A., \& Crawley, E. (2020). Rapid systematic review: the impact of social isolation and loneliness on the mental health of children and adolescents in the context of COVID-19. Journal of the American Academy of Child and Adolescent Psychiatry, S0890-8567(0820), 30337-30333. https://doi.org/10. 1016/j.jaac.2020.05.009.

Manczak, E. M., Ordaz, S. J., Singh, M. K., Goyer, M. S., \& Gotlib, I. H. (2019). Time spent with parents predicts change in depressive symptoms in adolescents with major depressive disorder. Journal of Abnormal Child Psychology, 47(8), 1401-1408. https://doi. org/10.1007/s10802-019-00526-5.

Meuwese, R., Cillessen, A. H. N., \& Güroğlu, B. (2017). Friends in high places: a dyadic perspective on peer status as predictor of friendship quality and the mediating role of empathy and prosocial behavior. Social Development, 26(3), 503-519. https://doi. org/10.1111/sode.12213.

Montoya, A. K. (2019). Moderation analysis in two-instance repeated measures designs: probing methods and multiple moderator models. Behavior Research Methods, 51(1), 61-82. https://doi. org/10.3758/s13428-018-1088-6.

Montoya, A. K., \& Hayes, A. F. (2017). Two-condition within-participant statistical mediation analysis: a path-analytic framework. Psychological Methods, 22(1), 6. 
Pappa, S., Ntella, V., Giannakas, T., Giannakoulis, V. G., Papoutsi, E., $\&$ Katsaounou, P. (2020). Prevalence of depression, anxiety, and insomnia among healthcare workers during the COVID-19 pandemic: a systematic review and meta-analysis. Brain, Behavior, and Immunity, 88, 901-907. https://doi.org/10.1016/j.bbi.2020. 05.026.

Rapee, R. M., Oar, E. L., Johnco, C. J., Forbes, M. K., Fardouly, J., Magson, N. R., \& Richardson, C. E. (2019). Adolescent development and risk for the onset of social-emotional disorders: a review and conceptual model. Behaviour Research and Therapy, 123, 103501. https://doi.org/10.1016/j.brat.2019.103501.

Richardson, C. E., Magson, N. R., Fardouly, J., Oar, E. L., Forbes, M. K., Johnco, C. J., \& Rapee, R. M. (2020). Longitudinal associations between coping strategies and psychopathology in preadolescence. Journal of Youth and Adolescence. (in press).

Rose, A. J., \& Rudolph, K. D. (2006). A review of sex differences in peer relationship processes: potential trade-offs for the emotional and behavioral development of girls and boys. Psychological Bulletin, 132(1), 98.

Somerville, L. H. (2013). The teenage brain: sensitivity to social evaluation. Current Directions in Psychological Science, 22(2), 121-127.

Somerville, L. H., Jones, R. M., \& Casey, B. (2010). A time of change: behavioral and neural correlates of adolescent sensitivity to appetitive and aversive environmental cues. Brain and Cognition, 72(1), 124-133.

Spence, S. H. (1998). A measure of anxiety symptoms among children. Behaviour Research and Therapy, 36(5), 545-566.

Tamres, L. K., Janicki, D., \& Helgeson, V. S. (2002). Sex differences in coping behavior: a meta-analytic review and an examination of relative coping. Personality and Social Psychology Review, 6(1), 2-30. https://doi.org/10.1207/s15327957pspr0601_1.

Wang, C., Pan, R., Wan, X., Tan, Y., Xu, L., Ho, C. S., \& Ho, R. C. (2020). Immediate psychological responses and associated factors during the initial stage of the 2019 coronavirus disease (COVID19) epidemic among the general population in China. International Journal of Environmental Research and Public Health, 17 (5), 1729.

Wang, C., Pan, R., Wan, X., Tan, Y., Xu, L., McIntyre, R. S., ... Sharma, V. K. (2020). A longitudinal study on the mental health of general population during the COVID-19 epidemic in China. Brain, Behavior, and Immunity.

Natasha R. Magson is a Postdoctoral Research Fellow at the Centre for Emotional Health, Macquarie University, Australia. Her research interests include peer influences on adolescent mental health and the impact of peer victimization and weight stigma on adolescent wellbeing.

Justin Y. A. Freeman is a project officer at in the Department of Psychology at Macquarie University. He has extensive experience running large scale longitudinal projects with adolescents and their families, and has a strong interest in adolescent mental health and associated influencing factors.

Ronald M. Rapee is a Distinguished Professor at the Centre for Emotional Health, Macquarie University, Australia. His research specializes in mental health, especially in anxiety and related disorders across the lifespan and he is best known for his theoretical models of the development of anxiety disorders as well as for his creation of empirically validated intervention programs.

Cele E. Richardson is a Lecturer in the School of Psychological Science and Centre for Sleep Science at The University of Western Australia. Cele is also an honorary lecturer at the Centre for Emotional Health, Macquarie University and her research interests include adolescent sleep and mental health.

Ella L. Oar is a Postdoctoral Research Fellow at the Centre for Emotional Health, Macquarie University, Australia. Her research aims to better understand the factors underlying the development of anxiety and obsessive-compulsive disorders in addition to enhancing treatment outcomes and access for youth who suffer from these debilitating conditions.

Jasmine Fardouly is a Postdoctoral Research Fellow at the Centre for Emotional Health, Macquarie University, Australia. Her research interests include social influences on the mental health of young people, particularly the influence of social media use. 